\title{
En el camino del EEES: La competencia del inglés en los universitarios de empresa. Un análisis en la Universidad de Jaén
}

On the way to the EHEA: English competence in business studies. An analysis at the University of Jaén

Francisca Castilla Polo (fpolo@ujaen.es)

Alonso Moreno Aguayo (alonso.moreno@ujaen.es)

Macario Cámara de la Fuente (mcamara@ujaen.es)

Eva $M^{a}$ Chamorro Rufián (echamo@ujaen.es)

Universidad de Jaén (España)

http://dx.doi.org/10.12795/EDUCADE.2012.i03.03

RESUMEN: De las novedades más significativas que conlleva el Espacio Europeo de Educación Superior (EEES), es destacable un modelo de aprendizaje que atribuye su éxito o fracaso en virtud de la adquisición o no de determinadas competencias. Junto a las competencias específicas de cada titulación, encontramos otras que adquieren un carácter genérico o transversal. De ellas, resulta especialmente relevante el caso de la competencia idiomática, o segundo idioma, por sus implicaciones tanto para el alumno como para el docente universitario.

Hemos revisado el estado del conocimiento y la utilidad que ambos colectivos le atribuyen para un caso concreto, el de los estudiantes de Administración y Dirección de Empresas (ADE) de la Universidad de Jaén, y hemos encontrado que efectivamente existe una brecha entre los planteamientos teóricos sobre el nivel que para ambos grupos sería deseable en esta competencia y los hallados en la realidad analizada. La metodología utilizada ha consistido en la realización de encuestas en un primer momento y en la posterior triangulación de la información obtenida a través de dos actividades presenciales con los alumnos sobre este tema.

Las conclusiones de nuestra investigación pueden ser especialmente útiles para los dirigentes universitarios que tienen entre sus responsabilidades el organizar las actividades necesarias para la adquisición de las habilidades a las que el nuevo sistema universitario obliga.

PALABRAS CLAVE: Competencias, Administración y Dirección de Empresas, Espacio Europeo de Educación Superior.

ABSTRACT: The most significant innovation that comes as a direct result from the European Higher Education Area (EHEA) is the learning model that is attached to its success or failure in virtue of the acquisition, or lack of it, in the determined competencies. Attached to the specific competencies for each one of the degrees, there are others which have a generic or transversal character. Especially relevant is the idiomatic competency, or second language, for its implications to the student and for the professor or instructor.

We have reviewed the knowledge estate and the utilization that both parts contribute to the specific case, this case takes into consideration the studies of Business Administration at the University of Jaén. The results from the study have found that in effect there is a significant gap between the theory and the level of performance by both groups. This is a conclusion that has come as a result of surveys and by triangulating the collected information and results of two analyses made in real life scenarios to the students.

Artículo. Recibido: 18-01-11 - Versiones revisadas: 29-06-11, Aceptado: 20-11-11

Licencia Creative Commons BY NC ND $\cdot 2012 \cdot$ Asociación Española de Contabilidad y Administración de Empresas - AECA 
The results from this study could be of significant help and assistance to the heads of the educational system that are in charge of developing the new programs and activities needed to acquire the abilities that the new Higher Education system has established.

KEYWORDS: Skills, Business Studies, European Higher Education Area.

\section{INTRODUCCIÓN}

Nos encontramos en un momento en el que la enseñanza universitaria no puede ignorar la importancia de un segundo idioma ni como materia de estudio ni como elemento educativo a utilizar en la formación de los ciudadanos. La incorporación a un EEES justifica aun más la necesidad del idioma como una competencia transversal que posibilite la movilidad de los estudiantes y que a la vez permita el acceso a recursos diferentes a los tradicionalmente empleados por el ámbito educativo español. Resultan continuas las demandas del actual modelo de educación en materia de idiomas, por entender que en los momentos actuales éste no se ha adaptado a la situación presente, con un sistema educativo como el que propone la Declaración de Bolonia, (1999) y Tudor, (2005).

En este sentido, encontramos de forma recurrente referencias a la importancia del conocimiento de idiomas, y de su inclusión como competencia transversal en algunos documentos que han sido básicos para la elaboración de los nuevos planes de estudios universitarios, como es el de los Libros Blancos de ANECA, concretamente para el caso que estudiamos el Libro Blanco para el Título de Grado en Economía y en Empresa (ANECA, 2005), en el que se recoge como primera competencia genérica, en el ámbito de los estudios de empresa, la que se obtiene con el conocimiento de los idiomas (p. 172). Allí mismo se señala como una de las competencias y habilidades que deben haber adquirido estos graduados, la de "leer y comunicarse en más de un idioma, en especial en inglés" (ANECA, 2005: 415).

Por otra parte, queremos recoger como la Asociación de las Universidades Públicas de Andalucía (AUPA), en 2008, de entre los requisitos y condiciones establecidas para la implantación del EEES, acordó la necesidad del conocimiento de idiomas, que será "una competencia transversal que debería incluirse y exigirse en todas las titulaciones a nivel andaluz...; y el alumnado tendrá que acreditar al finalizar su titulación el conocimiento de un segundo idioma" (AUPA, 2008).

El conocimiento y la utilización de un segundo idioma, y más concretamente del inglés, adquieren una relevancia máxima en el contexto del alumnado universitario (European Language Council, 2001). Se pretende la movilidad y el intercambio de nuestros alumnos entre, como mínimo, los países miembros de la UE, y esto significa que un segundo idioma es una competencia necesaria, toda una oportunidad para ampliar sus horizontes geográficos y educativos. Si bien no sólo resulta competencia del alumnado el dominio de una segunda lengua, ya que también lo es para el docente como segunda parte implicada en el proceso de aprendizaje. A él le corresponde la labor no sólo de poseer la competencia de un segundo idioma, inglés de manera general, sino también la de motivar su adquisición en su alumnado. Son muchas ya las universidades que ofertan alguna parte de su docencia en inglés, con una cada vez mejor acogida por parte de los estudiantes, siendo uno de los indicadores reales del prestigio y atractivo de las mismas, que otorga un gran valor.

En definitiva, entendemos que el conocimiento de una segunda lengua, especialmente el inglés como idioma internacional, es hoy en día una necesidad, no 
sólo en el ámbito académico y científico, sino también en el mundo de los negocios, en una economía global en las que sus relaciones económicas y financieras tienen que apoyarse en una comunicación en un lenguaje común, y no cabe duda que es el inglés la lengua franca de todo ese tipo de actividades; ello hace que sea una condición exigida en la carrera profesional de los gestores empresariales, quienes además van acompañados en su labor de nuevas tecnologías de la información y comunicación, en las que parte de sus herramientas son difíciles de manejar en una lengua distinta a la inglesa. Creemos que los docentes tenemos un reto importante para motivar a los alumnos en esta tarea, y que, aunque en los últimos años en España se haya producido un avance significativo en el reconocimiento de la importancia del dominio de otros idiomas, tradicionalmente ha sido una de las competencias menos conseguida por esta sociedad.

Han sido realizados ya algunos trabajos, al amparo de proyectos de innovación docente, sobre la transcendencia e importancia del conocimiento de idiomas en la nueva formación universitaria (Gallardo et alt, 2008; Corchuelo et alt., 2010). En este sentido, abordamos el presente trabajo con la finalidad de conocer el estado de esta competencia idiomática dentro del alumnado y profesorado de ADE de la Universidad de Jaén. Los resultados serán de una gran utilidad para los responsables universitarios a la hora de planificar las actividades docentes de las nuevas titulaciones de grado en las que es necesaria la acreditación del conocimiento del inglés como una de las habilidades más reconocidas y valoradas en el mundo laboral.

\section{OBJETIVOS}

De manera más específica, el presente trabajo pretende cubrir los siguientes objetivos:

- Conocer el nivel de dominio del idioma inglés que el alumno de la titulación de ADE manifiesta, y conocer a la vez la importancia que le atribuye. No sólo es interesante analizar la situación idiomática de partida del alumno sino su predisposición hacia la mejora y, específicamente, la utilidad que para su formación atribuyen a esta competencia.

- Conocer el nivel de dominio del idioma inglés que el profesorado de la titulación de ADE posee. Como responsable de la docencia, la figura del profesor y sus conocimientos en esta competencia consideramos que resultan básicos para la configuración del EEES. Por ello, nos preocuparemos por conocer dos aspectos específicos: a) nivel de dominio del propio profesor; y b) utilidad que atribuye a un buen conocimiento de un segundo idioma para su docencia universitaria.

- Elaborar propuestas para el desarrollo de la competencia idiomática en el alumnado y profesorado de la titulación de ADE. Sospechamos que existe una brecha entre los planteamientos teóricos que atribuye el EEES al alumnado y profesorado en materia de idiomas extranjeros y la realidad existente; no obstante deberemos comprobarlo. En caso positivo, nos proponemos analizar qué medidas podrían ser planteadas para llegar a acercar el nivel de dominio encontrado al que sería deseable, para que alumnos y profesores pudieran participar plenamente en el proceso de movilidad que para refuerzo de la competitividad de nuestro sistema universitario se le atribuye.

\section{METODOLOGÍA}

Dentro de la metodología utilizada para el desarrollo de este trabajo tenemos que destacar las siguientes fases realizadas con cada uno de los colectivos a los que nos hemos dirigido en nuestro análisis, y que son los que conforman el proceso de enseñanza-aprendizaje: alumnos y profesores. 


\subsection{Alumnos}

- Elaboración y distribución de un cuestionario a alumnos de ADE para el conocimiento de su nivel de idioma inglés así como para conocer la trascendencia de su mejora, en caso de ser necesaria. El cuestionario fue distribuido en los siguientes cursos y asignaturas (mayo de 2009): Economía de la Empresa I, primer curso; Contabilidad de Gestión I, segundo curso; Auditoría, tercer curso; y Dirección Estratégica II, cuarto curso.

La idea era recoger una muestra representativa de todos los cursos que integran el Plan de Estudios de esta titulación y, por extensión, de las cuestiones tratadas en el cuestionario sobre dominio, necesidad de mejora y alcance de la importancia de este idioma para su proceso de formación académica. En total fueron 329 los cuestionados obtenidos, de los estudiantes asistentes a esas clases.

- Focus-group para evidenciar el conocimiento de inglés por parte de los alumnos encuestados, que consistió en la impartición de una conferencia en inglés (mayo 2009), que sirvió para triangular la información obtenida de la encuesta previa, ya que, justo después de esta actividad, se les volvió a preguntar sobre algunos de los apartados a los que anteriormente ya habían respondido, y además por el propio contenido de la conferencia con el objetivo de comprobar cuál había sido el aprovechamiento y la comprensión sobre la lección impartida.

- Seminario con el objetivo de motivar a los alumnos sobre el aprendizaje y la utilidad del idioma inglés (mayo 2010). Para ello se contó con un académico y gestor universitario y un ejecutivo de la empresa privada. El primero transmitió la importancia de aprender idiomas y la necesidad actual de una acreditación sobre los mismos, además del valor añadido que supone la movilidad internacional, para llegar al mercado de trabajo en las mejores condiciones laborales. El segundo comenzó justo en este punto, explicando por su experiencia el valor competitivo que le ha otorgado el conocimiento de idiomas para escalar en su carrera profesional, y la importancia que ello tiene en las relaciones profesionales actuales.

\subsection{PROFESORES}

- Elaboración y distribución de un cuestionario a los profesores de ADE para conocer el estado y la importancia que concedían a su nivel de idioma inglés. En total se enviaron 66 cuestionarios por email, y también en papel (noviembre 2009), de los que se recibieron cumplimentados un total de 26 , lo que determina una tasa de respuesta del $39 \%$.

La elaboración de ambos cuestionarios tanto a alumnos como a profesores se basó en la búsqueda de respuesta a los objetivos de esta investigación así como en los referentes bibliográficos encontrados para cada caso. Ambos cuestionarios combinan una doble escala de medición. Por una parte, existen respuestas valorables según una escala Likert, generalmente relativas a situaciones deseables de esta competencia y opiniones personales sobre vías para su mejora, y por otro lado también aparecen otras cuestiones que se responden con una escala dicotómica para analizar la situación presente en esta competencia.

\section{RESULTADOS}

Dedicamos este apartado al comentario de los principales resultados obtenidos en nuestro estudio. Como se han identificado dos colectivos fundamentales: alumnos y profesores de $\mathrm{ADE}$, será cada uno de ellos objeto de un apartado específico que pasamos a comentar. 


\subsection{ResUltados Relativos a los alUmnos}

Respecto a los alumnos, presentamos en primer lugar los resultados del cuestionario que cumplimentaron en clase, en segundo lugar la evaluación a través de un focusgroup y una conferencia donde se les transmitió la importancia del inglés, y por último hacemos un comentario sobre el seminario de motivación.

\subsubsection{Resultados de la encuesta a los alumnos}

La distribución de los alumnos encuestados en los distintos cursos se muestra en la Tabla 1:

TABLA 1. Distribución de los ALUMnOS enCUestados (en PORCENTAJE)

\begin{tabular}{|l|c|}
\hline Primer curso & 41,3 \\
\hline Segundo curso & 32,0 \\
\hline Tercer curso & 14,6 \\
\hline Cuarto curso & 12,1 \\
\hline
\end{tabular}

1. Número de lenguas conocidas distintas a la materna. Todos los alumnos encuestados manifestaron conocer al menos una lengua distinta a la materna (media: 1,96). De forma concreta, el 12,2\% manifestó conocer sólo una lengua adicional, el $80,2 \%$ afirmó conocer dos lenguas adicionales, el 7,3\% respondió conocer tres lenguas adicionales, y el 0,3\% respondió conocer cuatro lenguas distintas a la materna. Respecto a las lenguas que son conocidas por los alumnos el $99,4 \%$ afirmó conocer inglés, el $84,8 \%$ francés, el 2,4\% alemán, mientras que el $4,3 \%$ afirmó conocer italiano. Otro 4,3\% afirmó conocer alguna otra lengua distinta a las anteriores, como portugués, árabe, polaco, ruso, ucraniano, chino, catalán y castellano, en este último caso, para alumnos extranjeros matriculados en la Universidad de Jaén y alumnos Erasmus.

2. Tiempo dedicado al aprendizaje de inglés, aparte de la enseñanza obligatoria (primaria y secundaria). Un 35,6\% afirmó no haber estudiado inglés más allá de la enseñanza obligatoria. Un $25,5 \%$ de los alumnos manifestó haber estudiado inglés por cuenta propia, con una media de 3,1 años; un 36,2\% afirmó haber estudiado inglés en una academia de idiomas, con una media de 3,6 años; un 13,7\% respondió haber estudiado inglés en una Escuela Oficial de Idiomas, habiendo dedicado una media de 2,5 años, mientras que un $4,9 \%$ afirmó haber estudiado inglés por otros medios, con una media de 0,9 años, como por ejemplo, a través de estancias en el extranjero.

3. Actividad de movilidad en el extranjero. Un 3,6\% de los alumnos encuestados afirmó haber realizado estudios preuniversitarios en el extranjero, con una media de 3,3 meses, habiéndose realizado el $41,7 \%$ de las mismas en Reino Unido; un 18,2\% aseguró haber realizado estancias de verano en el extranjero, con una media de un mes, de las cuales el $67 \%$ se realizaron en Reino Unido; un 3,6\% señaló haber realizado una estancia de movilidad dentro del programa Erasmus, con una media de 8,5 meses, habiendo en este caso tres países que comparten la primera posición, con un $25 \%$ cada uno: Italia, Portugal y Polonia, siendo este último el único donde el lenguaje utilizado en el centro destino es inglés; y un 6,7\% afirmó haber realizado otro tipo de movilidad internacional, como intercambios, viajes o campamentos, con una media de 1,1 mes, y en este caso principalmente a Francia, Italia, y en un segundo lugar a Reino Unido y Estados Unidos. Por último, a los que no habían realizado ninguna estancia en el extranjero, se les cuestionó sobre cuáles eran los motivos, alegando el $25,2 \%$ razones de tipo familiar/personal, el $23,4 \%$ insuficiente nivel de inglés, el $18,5 \%$ motivos de tipo económico, el 5,2\% estima no considerarlo necesario, y un $11,6 \%$ alega otros motivos distintos, como no haber tenido ocasión, no habérselo planteado o tenerlo previsto para años posteriores. 
4. Certificado de reconocimiento del nivel de inglés. Respecto a la posesión de un certificado oficial sobre reconocimiento del nivel de inglés de los alumnos, un 16,1\% aseveró tener algún certificado, mientras que el porcentaje restante $(83,9 \%)$ no posee ninguna acreditación al respecto. El desglose de las respuestas positivas es el siguiente: un 5,5\% aseguró ostentar el Certificado de la Escuela Oficial de Idiomas correspondiente al nivel intermedio (3er curso); un 3,6\% era portador del Cambridge First Certificate; un $0,3 \%$ era poseedor del Cambridge Advanced Certificate, ninguno poseía el Certificado de la Escuela Oficial de Idiomas correspondiente al nivel superior ( $5^{\circ}$ curso), ni el Cambridge Proficiency Certificate, ni había realizado el TOEFL. El 6,7\% afirmó poseer otros títulos, por cursos de verano en el extranjero o de otras universidades extranjeras.

5. Dominio autopercibido de la lengua inglesa. A los alumnos se les preguntó sobre el nivel de lectura, escritura y conversación que se atribuían respecto al dominio del inglés, debiendo situar su nivel entre muy bajo, bajo, medio, alto y muy alto. Mostramos los resultados en la Tabla 2.

TABLA 2. DOMINIO AUTOPERCIBIDO DE LA LENGUA INGLESA DE LOS ALUMNOS (EN PORCENTAJE)

\begin{tabular}{|l|l|l|l|l|l|}
\hline & Muy bajo & Bajo & Medio & Alto & $\begin{array}{l}\text { Muy } \\
\text { alto }\end{array}$ \\
\hline Lectura & 5,2 & 21,0 & 57,6 & 14,6 & 1,5 \\
\hline Escritura & 5,5 & 26,1 & 56,7 & 11,0 & 0,6 \\
\hline Conversación & 22,4 & 44,2 & 28,2 & 4,6 & 0,6 \\
\hline
\end{tabular}

6. Capacidad para realizar distintas actividades en inglés. Se preguntó a los alumnos sobre si se sentían capaces de realizar distintas tareas en inglés, como la preparación de un trabajo corto (resolución de un caso, opinión justificada sobre un tema, etc.), la exposición de un trabajo corto ayudado de un soporte informático (powerpoint o similar), el seguimiento de una exposición realizada por otros compañeros en clase, el seguimiento de una clase impartida por un profesor y la lectura y comprensión de bibliografía facilitada por el profesor, por tratarse de situaciones cercanas al uso del inglés académico.

Las respuestas disponibles para el alumno eran: afirmar que no era capaz o, en caso de una respuesta afirmativa, debía indicar el grado de calidad esperada (muy baja, baja, media, alta o muy alta). Se muestran los resultados en la Tabla 3.

7. Necesidad de mejora del inglés. El $97,8 \%$ de los alumnos encuestados reconocen su necesidad de mejorar su dominio del inglés. De ellos, el 1,3\% estima que el nivel de mejora necesario es bajo; el 29,3\% estima la necesidad de mejora en un nivel medio; y el $69,4 \%$ estima necesario un grado de mejora elevado. A estos mismos alumnos también se les cuestionó acerca del modo más oportuno en que abordaría esa necesidad de mejora.

Entre los procedimientos propuestos, las estancias en el extranjero ocupan el primer Iugar, con un 76,3\%; las Escuelas Oficiales de Idiomas ocupan el segundo lugar, con un 48,6\%; posteriormente se encontrarían las academias de idiomas, con un 34\%; y el estudio por cuenta propia, con un 14,6\%. Otros métodos distintos fueron referidos en un $4,6 \%$ de los casos, entre los que destaca la impartición en inglés de asignaturas del plan de estudios oficial de la titulación. 
Francisca Castilla, Alonso Moreno, Macario Cámara, Eva M Chamorro Competencia del inglés en los universitarios de empresa

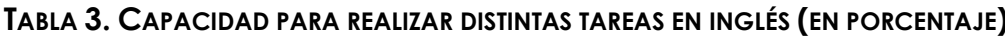

\begin{tabular}{|l|c|c|c|c|c|c|}
\hline & Nada & $\begin{array}{c}\text { Muy } \\
\text { baja }\end{array}$ & Baja & Media & Alta & $\begin{array}{c}\text { Muy } \\
\text { alta }\end{array}$ \\
\hline Preparación de un trabajo corto & 16,2 & 7,9 & 22,9 & 44,5 & 8,2 & 0,3 \\
\hline Presentación de un trabajo corto & 43,4 & 7,3 & 19,9 & 21,7 & 7,6 & 0,0 \\
\hline $\begin{array}{l}\text { Comprensión de una presentación } \\
\text { de un compañero }\end{array}$ & 23,9 & 12,6 & 23,9 & 29,8 & 9,2 & 0,6 \\
\hline $\begin{array}{l}\text { Comprensión de una clase } \\
\text { impartida por el profesor }\end{array}$ & 32,4 & 11,6 & 24,2 & 24,8 & 6,1 & 0,9 \\
\hline $\begin{array}{l}\text { Lectura y comprensión de } \\
\text { bibliografía }\end{array}$ & 30,0 & 9,5 & 22,9 & 26,3 & 10,7 & 0,6 \\
\hline
\end{tabular}

8. Importancia del inglés en el mercado laboral. Se preguntó a los alumnos sobre qué importancia atribuían al dominio del inglés en la mejora de sus oportunidades laborales. Además se les pidió opinión sobre esta importancia para el acceso a un puesto de trabajo en entidades públicas, en empresas privadas y en universidades. Los alumnos debían graduar la importancia en: muy baja, baja, media, alta o muy alta. Se muestran los resultados en la Tabla 4.

TABLA 4. IMPORTANCIA ATRIBUIDA POR LOS ALUMNOS AL INGLÉS EN El MERCADO LABORAL (EN PORCENTAJE)

\begin{tabular}{|l|c|c|c|c|c|}
\hline & $\begin{array}{c}\text { Muy } \\
\text { baja }\end{array}$ & Baja & Media & Alta & $\begin{array}{c}\text { Muy } \\
\text { alta }\end{array}$ \\
\hline Para mejorar las oportunidades laborales & 0,6 & 11,8 & 27,4 & 34,9 & 25,2 \\
\hline Para trabajar en el sector público & 0,6 & 11,8 & 27,4 & 34,9 & 25,2 \\
\hline Para trabajar en el sector privado & 0,0 & 2,8 & 11,3 & 38,8 & 47,2 \\
\hline Para trabajar en universidades & 0,3 & 3,8 & 26,9 & 42,8 & 26,3 \\
\hline
\end{tabular}

9. Presencia del inglés en la formación universitaria. Se les preguntó a los alumnos sobre si estimaban oportuno que el idioma inglés estuviese presente en la formación universitaria. El 90\% respondió afirmativamente, y por tanto, se les pidió que graduasen la necesidad de su incorporación (muy alta, baja, regular, alta o muy alta). Los resultados se muestran en la Tabla 5.

TAbla 5. Presencia del inglés en la formación universitaria (en porCentaje)

\begin{tabular}{|l|c|c|c|c|c|c|}
\hline & Nada & $\begin{array}{c}\text { Muy } \\
\text { baja }\end{array}$ & Baja & Media & Alta & $\begin{array}{c}\text { Muy } \\
\text { alta }\end{array}$ \\
\hline $\begin{array}{l}\text { Necesidad de incorporar el idioma } \\
\text { inglés en la formación universitaria }\end{array}$ & 8,4 & 0,3 & 1,2 & 16,8 & 44,1 & 29,2 \\
\hline $\begin{array}{l}\text { Importancia de contar con } \\
\text { asignaturas en inglés en la } \\
\text { formación universitaria }\end{array}$ & - & 1,6 & 4,1 & 19,4 & 49,1 & 25,9 \\
\hline
\end{tabular}

Del mismo modo, se les preguntó directamente si estarían interesados en recibir alguna asignatura en inglés. El 76,8\% respondió afirmativamente y el 23,2\% no mostró interés en matricularse en dichas asignaturas. Además, para ambas respuestas, se les pidió que las razonaran. Así, entre los alumnos que sí mostraron interés destaca el argumento mayoritario de que sería muy útil para su futuro laboral, y entre los que no mostraron interés en asignaturas en inglés, aún reconociendo la mayoría su importancia, argumentaron su respuesta incidiendo en las dificultades que ello le supondría para superar la asignatura debido a su bajo nivel inicial del mismo. Además, a los que respondieron afirmativamente, se les pidió que indicasen su preferencia sobre el carácter de las asignaturas en el que estarían más interesados que se impartiese en inglés, a lo que el $40,3 \%$ respondió que en una asignatura obligatoria y el $59,7 \%$ que en 
una asignatura optativa. Para terminar, se solicitó a los alumnos que graduaran la importancia de contar en su proceso de formación con una asignatura de este tipo. Las respuestas se muestran en la Tabla 5.

\subsubsection{Evidencia a través de un focus-group con los alumnos}

Una vez que habíamos obtenido y analizado los cuestionarios de los alumnos, nos planteamos verificar si el grado de conocimiento del inglés expresado por ellos, coincidía con su nivel real, con lo que nos propusimos medir el grado de comprensión real de una muestra de los alumnos previamente encuestados. Para ello, a través de una clase en inglés impartida en el aula pretendíamos corroborar con evidencias empíricas las indicaciones ofrecidas por los alumnos de ADE en cuanto a su nivel de inglés.

La técnica utilizada para alcanzar el objetivo anterior consistió en la realización de un seminario en inglés dirigido a los alumnos participantes en nuestro proyecto de innovación docente. Para tal fin, aprovechamos la visita docente del profesor Bradley Braun, de la University of Central Florida realizada durante el mes de mayo de 2009 a la Universidad de Jaén.

Concretamente, se organizó un seminario en el Salón de Grados de nuestra Universidad el día 7 de mayo titulado "Global Economics", de dos horas de duración, que fue impartido por Bradley Braun en el que se trataron fundamentalmente aspectos relacionados con el comercio internacional, tema presumiblemente de interés y relación para el alumno de $A D E$, y por supuesto en idioma inglés, para analizar con posterioridad el grado de asimilación de contenidos.

Una parte de los alumnos de ADE que habían participado previamente en este proyecto de innovación docente a través de la cumplimentación del cuestionario sobre la importancia del idioma inglés, asistieron voluntariamente a este seminario, recibiendo como reconocimiento del interés mostrado hacia esta segunda actividad un certificado de colaboración. Concretamente asistieron 31 alumnos: 20 de tercer curso y 11 de segundo curso.

Una vez finalizada la clase, se pidió a los alumnos que completasen un cuestionario con dos bloques. El primero, sobre el grado del entendimiento de la clase, además de su necesidad de mejora estimada en lectura, escritura y conversación en el idioma inglés. La Tabla 6 muestra los resultados.

TABLA 6. Comprensión de CLASE y NeCESidAd DE MEJORA (EN PORCENTAJE)

\begin{tabular}{|l|c|c|c|c|c|c|}
\hline & Nada & $\begin{array}{c}\text { Muy } \\
\text { baja }\end{array}$ & Baja & Media & Alta & $\begin{array}{c}\text { Muy } \\
\text { alta }\end{array}$ \\
\hline Comprensión de la clase & 6,5 & 16,1 & 25,8 & 12,9 & 29,0 & 9,7 \\
\hline Necesidad de mejora en lectura & 0,0 & 3,3 & 16,7 & 43,3 & 23,3 & 13,3 \\
\hline Necesidad de mejora en escritura & 0,0 & 0,0 & 6,7 & 60,0 & 20,0 & 13,3 \\
\hline $\begin{array}{l}\text { Necesidad de mejora en } \\
\text { conversación }\end{array}$ & 0,0 & 3,3 & 20,0 & 13,3 & 43,3 & 20,0 \\
\hline
\end{tabular}

Asimismo, en un segundo bloque, se solicitó a los alumnos de ADE que rellenaran un test de asimilación de contenidos consistente en siete preguntas, valoradas cada una de ellas con un punto si era contestada correctamente, es decir, el test tenía una valoración máxima de 7 puntos. Se trataba de preguntas con respuestas verdadero o falso, dos de ellas, así como también se incorporaron cinco preguntas cortas. La puntuación media de los 31 alumnos examinados fue de 2,4 (sobre un máximo de 7). No obstante, queremos dejar constancia como aparece en el gráfico sobre 
entendimiento de la clase, que el 38,7\% manifestó haber comprendido el contenido de la charla con un nivel alto o muy alto.

\subsubsection{SEMINARIO DE MOTIVACIÓN SOBRE LA IMPORTANCIA DEL INGLÉS}

Como fase final del proyecto, se invitó a dos personas que por su trayectoria profesional pudieran transmitir a los alumnos de ADE, de las asignaturas Auditoría y Contabilidad de Gestión I, el valor y la importancia que en la sociedad y la economía actual tiene el conocimiento de idiomas, como ventaja competitiva en la actividad profesional de los titulados universitarios especialistas en empresa. Los dos ponentes, Sebastián Bruque' y Pedro Vilches², hicieron sus presentaciones el día 19 de mayo de 2010, de forma sucesiva, y a saber por los comentarios posteriores que los alumnos le hicieron a los responsables de este proyecto de innovación docente se sintieron muy motivados en la adquisición de esta competencia, y valoraron muy satisfactoriamente la elección de los intervinientes por su capacidad y sinceridad para transmitir la necesidad del conocimiento de idiomas -especialmente inglés- para encontrar un empleo satisfactorio.

Sebastián Bruque se centró en la importancia del inglés dentro del propio proceso educativo, en este caso dentro de la Universidad de Jaén, y en su presentación fue recurrente sobre la necesidad de que cuando sean egresados universitarios tengan un conocimiento suficiente $-y$, si es posible, acreditado- para incorporarse al mercado laboral con una mayor potencialidad para encontrar una ocupación acorde con los competencias específicas adquiridas en su carrera.

Comienza su charla con un análisis de los principales idiomas utilizados en la educación superior en el mundo, y su extensión y grado de utilización, para llegar a establecer la importancia del inglés como instrumento de comunicación en la educación superior, y como lengua franca y científica. Otras referencias significativas en esta primera parte de su intervención estuvieron dedicadas a la evolución y a la importancia de la movilidad estudiantil en los últimos años, y los valiosos resultados conseguidos con esta actividad por parte de los alumnos, que tan positivamente vienen valorando los empleadores. Aprovechó en este sentido para recordar a los alumnos el sistema de movilidad internacional que tiene establecido la Universidad de Jaén: convocatorias, requisitos, tipos de ayudas, etc., así como la organización de cursos de idiomas dirigidos a la comunidad universitaria, y las expectativas del Centro de Estudios Avanzados en Lenguas Modernas de la Universidad de Jaén.

La segunda parte (la más extensa) la dedicó a explicar los distintos sistemas, más generalmente aceptados, de acreditación y reconocimiento del idioma inglés en la educación superior y en la movilidad Internacional; estos son: El Marco Común Europeo de Referencia para las Lenguas, la escala de Cambridge, la escala IELTS y la escala TOEFL/TOEIC. Se detalla la aplicabilidad de cada uno según entornos, se establecen las equivalencias entre los distintos sistemas, y se señalan cuáles son los niveles admitidos en las universidades extranjeras para grado y posgrado. Concluye

\footnotetext{
I Sebastián Bruque es Profesor Titular del área de Organización de Empresas de la Universidad de Jaén, y actualmente ocupa el cargo de Director del Secretariado de Relaciones Internacionales de esta Universidad. De él dependen los programas de movilidad internacional, y su Vicerrectorado es el encargado de la organización de las enseñanzas de idiomas en la Universidad de Jaén, como competencia transversal en los títulos oficiales distintos a los propios de lenguas.

2 Pedro Vilches es Licenciado en Ciencias Empresariales por la Universidad de Jaén, y en la actualidad es Director Financiero (Chief Financial Officer and IT) de Clartonhorn, SA, empresa sucesora de Robert Bosch España, SA, en La Carolina (Jaén).
} 
animando a la acreditación, ya que hoy en día es una exigencia, pero sin duda lo será todavía más en el futuro más inmediato, como consta en las propias memorias de los nuevos Grados, que obligan a demostrar (de forma acreditada) el conocimiento de idiomas.

Pedro Vilches inicia su presentación dirigiéndose a los alumnos en inglés y en alemán, en un intento de elevar, desde el primer momento, el interés de los alumnos sobre el idioma, para pasar a continuación a decirles que seguramente se encontrarán en muchas ocasiones con circunstancias como ésta, ya que ese va a ser el día a día en el ejercicio de su profesión. Continúa, después de poner el punto de atención en ello, explicando cuál es la actividad de su empresa, encargada del desarrollo, fabricación y venta de componentes de automoción, de carácter mundial.

La segunda parte de su intervención la centra en contar su experiencia, comenzando por las "prácticas en empresa" que realizó en Robert Bosch España, SA (Fábrica de La Carolina), que es donde acabó empleado en 1998. Ya en esos momentos sintió la necesidad de su aprendizaje de inglés, porque vio que la condición para poder conseguir un trabajo, en esta empresa, acorde con la titulación universitaria que poseía, era conocer (al menos) inglés a un nivel suficiente como para poder mantener con fluidez una conversación y poder comunicarse de forma escrita con un nivel también aceptable. Ello le llevó a conseguir esta capacidad, con sus propios recursos económicos, acudiendo a los mejores centros especializados en ese momento en su entorno más inmediato (Jaén y La Carolina). También hace una extensa referencia a la oportunidad que le ha brindado el conocimiento de inglés, no sólo en su vida profesional, sino también personal. Ello le sirve como línea argumental para plantear la necesidad de que cada uno establezca una estrategia de motivación personal para desarrollar su conocimiento de idiomas.

Basa una parte fundamental de su presentación en ejemplos reales y anécdotas sobre cómo el conocimiento del inglés le ha servido para solventar problemas o aprovechar las oportunidades que el mercado laboral y el entorno personal le brindaba. Asimismo propone a los alumnos que tengan en cuenta la situación actual de amenazas y oportunidades que ofrece el mercado laboral (y académico), así cómo se pueden aprovechar las fortalezas que el sistema tiene actualmente: la existencia de programas de movilidad estudiantil y de intercambio cultural, enseñanza del idioma, facilidad de acreditaciones; que como hemos comentado expuso el profesor Bruque. Termina llamando a la automotivación (como empezó), proponiendo romper las barreras internas y animando a la práctica del idioma, sin complejos, como base para evolucionar en su conocimiento.

\subsection{Resultados de la encuesta A los profesores}

Incorporamos en este bloque los principales resultados obtenidos a través del tratamiento estadístico de los cuestionarios recibidos por parte de los profesores de la titulación de ADE que han participado en nuestro estudio. Laboralmente, podemos afirmar que las categorías que más han colaborado son: Profesor Titular de Universidad, 33,3\%; Profesor Titular de Escuela Universitaria, 16,6\% y Profesor Contratado Doctor, 16,6\%. En cuanto a la titulación académica de los docentes, sobresale ampliamente la titulación de "Ciencias Empresariales, Económicas o Administración y Dirección de Empresas", 83,3\% de los casos, y la de "Estadística/Matemática", con un $12,5 \%$.

1. Número de lenguas conocidas distintas a la materna. Todos los profesores encuestados manifestaron conocer al menos una lengua diferente a la propia o materna. En un $60 \%$ de los casos, el docente conoce un solo idioma adicional: idioma inglés, siendo el $36 \%$ de los profesores conocedores de dos idiomas: inglés y francés, y 
sólo un caso, que representa el $4 \%$ del total, el único que admite manejar tres idiomas: inglés, francés y portugués. Resulta significativo que, en ningún caso conozcan el idioma italiano y alemán, a pesar de su relevancia.

2. Tiempo dedicado al aprendizaje de inglés, aparte de la enseñanza obligatoria (primaria y secundaria). El estudio de inglés por cuenta propia es la fórmula más utilizada por los profesores de ADE para mejorar el aprendizaje de la lengua inglesa, un $40 \%$ de estos lo han empleado y con una duración media de 5,35 años. Un $20 \%$ de los profesores manifestó haber estudiado inglés en una academia de idiomas, durante como media 3 años. Le sigue en orden de importancia el aprendizaje de inglés dentro de Escuelas Oficiales de Idiomas, un $16 \%$ y una duración media de 2,7 años. Otras opciones para el estudio de inglés utilizadas con carácter más residual son las estancias en el extranjero, $12 \%$, la asistencia a cursos ofertados por la Universidad de Jaén, $8 \%$, y la utilización de un profesor particular, $4 \%$. Ahora bien, el empleo de las diferentes vías de aprendizaje no resulta exclusiva, así en el $72 \%$ de los casos, el docente combina, al menos, dos formas diferentes de aprendizaje de este idioma.

3. Actividad de movilidad en el extranjero. Por las implicaciones de esta actividad en el EEES, se decidió incorporar una pregunta específica para conocer las vías, duración y lugares donde los profesores de ADE participantes en nuestro estudio han realizado alguna actividad de movilidad. La forma más utilizada ha sido las estancias de verano en el extranjero, en un $28 \%$ de los casos, siendo los destinos más elegidos Reino Unido $(66,7 \%)$ y EEUU $(33,3 \%)$. Le sigue con un $12 \%$ de los casos la movilidad docente bajo el programa Erasmus, Teaching Staff Mobility, donde ninguno de los destinos europeos sobresale sobre sus homólogos: Francia, Italia y Dinamarca. La realización de visitas docentes es otra vía utilizada para la movilidad, $12 \%$ de los casos y con un horizonte europeo en los destinos para el $100 \%$ de los casos: Francia $66,7 \%$ y Portugal $33,3 \%$ restante. Sobre las estancias pre y post doctorales, en países de la UE siempre, debemos indicar que se han realizado con la misma frecuencia, $8 \%$ de los casos, y que sólo en las últimas, las estancias postdoctorales, observamos que es posible indicar un destino más elegido: Italia. También se indica como otra vía de movilidad, la realización de viajes personales, $8 \%$ de los casos, y la asistencia a cursos y congresos internacionales, $8 \%$. La no realización de actividades de movilidad por los docentes estudiados se justifica por los siguientes motivos: de tipo familiar/personal (64,3\%), otros motivos (28,6\%), la falta de tiempo (16\%), motivos económicos $(14,3 \%)$ e insuficiente nivel de inglés $(7,1 \%)$. En ningún caso se planteó que la movilidad no fuese considerada necesaria por los profesores participantes así como también debemos comentar que la pregunta podía dar lugar a respuestas múltiples, es decir, a más de un motivo para justificar la falta de movilidad del docente.

4. Certificado de reconocimiento de nivel de inglés. Respecto a los certificados oficiales que avalan el nivel de inglés poseído por el profesor de ADE, debemos indicar que disponen de ellos un 36\%. Dentro de las categorías que más se repiten, debemos destacar: el Certificado de la Escuela Oficial de Idiomas (nivel intermedio), 55,6\%; el Certificado de la Escuela Oficial de Idiomas (nivel superior), 33,3\%; el Cambridge First Certificate, 33,3\%; el Cambridge Advanced Certificate, 11,1\%; y el TOEFL, 11,1\%. Sólo dos profesores manifestaron poseer más de un certificado oficial.

5. Dominio autopercibido de la lengua inglesa. Cuando se ha preguntado directamente por el dominio del docente de la lengua inglesa en sus tres vertientes: lectura, escritura y conversación, los resultados obtenidos son los que aparecen en la Tabla 7. Claramente podemos observar como varían según categoría de dominio, sobresaliendo la habilidad para la lectura de textos en lengua inglesa a diferencia del dominio para escribir y conversar en este idioma que aparecen con una valoración similar y más reducida. No obstante, el valor de la escala "muy alto" no ha aparecido en ningún caso. 
Francisca Castilla, Alonso Moreno, Macario Cámara, Eva $M^{a}$ Chamorro Competencia del inglés en los universitarios de empresa

TABLA 7. DOMINIO AUTOPERCIBIDO DE LA LENGUA INGLESA DE LOS PROFESORES (EN PORCENTAJE)

\begin{tabular}{|l|c|c|c|c|c|}
\hline & $\begin{array}{c}\text { Muy } \\
\text { bajo }\end{array}$ & Bajo & Medio & Alto & $\begin{array}{c}\text { Muy } \\
\text { alto }\end{array}$ \\
\hline Lectura & 4,2 & 4,2 & 37,5 & 54,2 & 0,0 \\
\hline Escritura & 8,3 & 29,2 & 41,7 & 20,8 & 0,0 \\
\hline Conversación & 12,5 & 25,0 & 45,8 & 16,7 & 0,0 \\
\hline
\end{tabular}

6. Capacidad para la docencia en inglés. Se preguntó al profesorado de ADE si sería capaz de exponer su docencia en inglés, apoyado por soporte informático para la presentación, y los resultados nos indican que un $62,5 \%$ serían capaces de tal actividad, esperando ellos mismos una calidad, dentro de una escala Likert (muy baja, baja, media, alta y muy alta), alta (20\%), media $(46,7 \%)$, baja $(26,7 \%)$ y muy baja $(6,7 \%)$. En ningún momento apareció el valor de la escala "muy alta". Lo anterior puede relacionarse con el hecho de que cuando se les preguntó por el interés, no la capacidad, de impartir docencia en lengua inglesa, los resultados obtenidos indican que tan sólo un $33,3 \%$ lo estaría.

Una iniciativa de la Universidad de Jaén, Vicerrectorado de Relaciones Internacionales y Cooperación, relacionada con la docencia en lengua inglesa, pero sólo para aquellos estudiantes participantes dentro del Programa Sócrates-Erasmus, es el Programa de Ayuda y Tutorización en Inglés al Estudiante Extranjero (PATIE). Los profesores participantes en el mismo puede elegir diferentes grados de apoyo a estos estudiantes que van desde un nivel 1, básico, hasta un nivel 3, avanzado, en el que el compromiso de apoyo docente en inglés es el más elevado. Cuando se interrogó a los profesores sobre su colaboración con este proyecto, los resultados encontrados muestran que sólo un $40 \%$ de ellos participan en él. De los cuales, el $60 \%$ con un nivel 1 o bajo, el $30 \%$ con un nivel medio y sólo un docente (10\%) participa en el nivel más avanzado. Fuera de este proyecto, la impartición de contenidos en inglés por los profesores estudiados es inexistente; sí se detecta cierto interés en el suministro de bibliografía en este idioma, en un $32 \%$.

7. Necesidad de mejora del inglés. El $96 \%$ de los profesores encuestados reconocen la necesidad de mejorar su dominio de la lengua inglesa. Cuando se solicitó en una escala Likert con tres valores (bajo, medio, alto) que indicasen el grado de mejora necesitado, los resultados obtenidos indican, en su gran mayoría, la necesidad de mejora que sería media, 56,5\%; alto, 39,1\%; y sólo en un caso, $4,3 \%$, el docente manifestó necesitar un grado bajo de mejora de su nivel de inglés. Sobre las vías o medios para efectuar esa mejora diagnosticada como necesaria por el profesor, sobresale en un $75 \%$ de los casos, la utilización de estancias en el extranjero para tal fin, seguidas del estudio por cuenta propia de este idioma, en un $58,3 \%$, la asistencia a Academias de Idiomas, en un $20,8 \%$ y Escuelas Oficiales de Idiomas, $16.6 \%$. También se indicó en el apartado "otros" que fuese la Universidad de Jaén la ofertante de estos cursos de mejora, así como la asistencia a clases particulares con un profesor nativo.

8. Importancia del inglés en la mejora de oportunidades laborales. Se incluyó en el cuestionario una pregunta valorada por el profesor a través de una escala Likert (muy bajo, bajo, medio, alto y muy alto), sobre la importancia que concedía al idioma inglés para mejorar sus oportunidades laborales. Los resultados son altamente positivos, encontrándonos que un $56 \%$ de los docentes consideran "muy alta" esta relevancia, seguida de un $36 \%$ de ellos que la califican como "alta". En ningún momento han aparecido los valores bajo y muy bajo de la escala, sí una importancia "media" para un $8 \%$ de los casos.

9. Presencia del inglés en la formación universitaria. Un último bloque del cuestionario se dedicó al análisis de la importancia que el profesor de ADE concede al hecho de que la formación universitaria incorporase el idioma inglés. En un 91,3\% de los casos se 
consideró necesaria tal incorporación, valorándose esta necesidad mayoritariamente como muy alta, 52,2\%; alta, 34,8\%; media, $8,7 \%$; y baja en un solo caso, $4,3 \%$. Cuando como caso concreto, se interrogó al docente sobre su interés en impartir alguna asignatura en lengua inglesa, los resultados muestran mayoritariamente que no lo consideran en este momento interesante, expresando motivos como los siguientes por orden de importancia: falta de conocimientos (40\%), falta de tiempo (8\%), demasiado esfuerzo en su preparación (8\%). Si bien, sí tienen claro que sería una asignatura optativa la que mejor se adaptaría al cambio de idioma propuesto, en un $81,8 \%$; $u$ obligatoria, en tan sólo un 18,2\%.

\section{CONCLUSIONES}

Con nuestro caso hemos intentado aportar evidencia sobre la importancia del idioma como competencia transversal en los estudios de ADE, y que creemos que es una habilidad necesaria en el ámbito académico. Para ello, hemos analizado el conocimiento real del idioma y lo hemos comparado con la importancia que le atribuyen los dos colectivos principalmente implicados en el proceso de aprendizaje: alumnos y profesores.

Tras analizar los resultados obtenidos en los cuestionarios realizados a alumnos podemos concluir, en relación con el primer objetivo, que el dominio de la lengua inglesa por los mismos se encuentra en un nivel medio-bajo. De las tres dimensiones analizadas: lectura, escritura y conversación, la última es la que presenta peores resultados en términos comparativos. No obstante, conceden una importancia considerable a la necesidad de mejora de su nivel, en un $97,8 \%$ de los casos, ya que entre otros motivos relacionan esta competencia con la mejora de sus oportunidades laborales en un grado muy alto, 63,1\%.

Sobre los resultados obtenidos por el colectivo de profesores de ADE que han participado en nuestro estudio, podemos concluir que, en general, el nivel de dominio de la lengua inglesa es más alto que en el colectivo de los alumnos. Igualmente, en un $96 \%$ de los casos, se considera necesario la mejora de este nivel en un grado medioalto. De forma más concreta y en cuanto al nivel de formación autopercibido por el docente para las tres dimensiones analizadas: lectura, escritura y conversación, vuelve a observarse que la conversación y la escritura quedan peor valoradas que la habilidad de leer textos en este idioma.

Si relacionamos los resultados obtenidos con la reciente incorporación al EEES, podemos concluir que todavía los colectivos analizados, y a la vez principales para su puesta en práctica, no parecen disponer de las habilidades suficientes en idioma. Hemos encontrado unas diferencias significativas entre el dominio real del idioma y la importancia que se le atribuye como competencia a lo largo de la carrera universitaria, por su oportunidad para el futuro profesional. Aunque algunos de los primeros datos obtenidos en el cuestionario parezcan evidenciar un conocimiento alto de otras lenguas, por parte de los alumnos (un $80,2 \%$ de ellos afirma conocer dos lenguas adicionales), y especialmente inglés (un 99,4\%), sin embargo, con el análisis de cuestiones posteriores, se pone de manifiesto que no es un dominio alto como para desenvolverse de forma exitosa, ya que consideran necesario mejorar su cualificación el $97,8 \%$ de los mismos. Para el colectivo de profesores, nos ha parecido muy significativo que, a pesar de que otorgan una gran importancia a la formación en idiomas de sus alumnos, consideran que: o bien no tienen la formación suficiente para impartir su docencia en inglés o bien pueden carecer de algún incentivo que compense el esfuerzo necesario para ello.

A través de la triangulación entre el cuestionario realizado y la evaluación sobre la lección impartida en inglés, podemos concluir que existe un importante gap entre la 
manifestación hecha sobre el conocimiento de inglés y lo realmente puesto de manifiesto a través de los valores conseguidos sobre comprensión del contenido de esta actividad, aunque queremos ponderar el hecho de que haya sido impartida esta conferencia por un nativo.

Los alumnos se sintieron muy animados y motivados para implicarse en el aprendizaje de inglés cuando recibieron información sobre la trascendencia de la acreditación de idiomas para poder llevar a cabo una actividad de movilidad estudiantil y para posteriormente trabajar a un nivel acorde con su titulación universitaria, así como la necesidad y oportunidad de poder comunicarse en inglés en las relaciones económicas y sociales.

\section{DISCUSIÓN}

A la vista de estos resultados consideramos que las universidades deben realizar importantes esfuerzos para reducir el gap entre la situación actual y la deseada para alumnos y profesores, para llegar realmente a una formación de calidad en el EEES. Estos pueden concretarse en actuaciones como las siguientes:

- Oferta de docencia en inglés, como estímulo a la necesidad de un nivel de inglés acorde con las necesidades del EEES. La Universidad de Jaén ya ha actuado en este sentido: i) en la Diplomatura en Ciencias Empresariales se imparte en inglés un grupo de prácticas de la asignatura Dirección Financiera; ii) la Facultad de Ciencias Sociales y Jurídicas ofrece dos asignaturas de libre configuración específica impartidas en inglés: International Commercial Law e International Management of Technology (la Universidad de Jaén, en el futuro inmediato, sólo ofertará asignaturas de libre configuración específica en inglés); iii) para los alumnos participantes en el programa Programa Sócrates-Erasmus está establecido el Programa de Ayuda y Tutorización en Inglés al Estudiante Extranjero (PATIE).

- Proyectos de innovación docente, como vías para fomentar iniciativas docentes que tengan en cuenta el papel del idioma inglés en la formación académica universitaria. Entre ellos podemos citar el que sirve de base para este trabajo, y otro que este mismo equipo investigador está iniciando "La movilidad internacional como medio práctico de mejora del inglés del alumnado universitario de Administración y Dirección de Empresas" (que ha sido concedido para el bienio 20102012). Este último tiene como objetivo reforzar el nivel de inglés del alumnado de ADE a través de una iniciativa de clases destinadas a estos estudiantes e impartidas por alumnos extranjeros procedentes de programas de movilidad internacional que se encuentren realizando una estancia en la Universidad de Jaén, con lo que se conseguirá satisfacer esta necesidad sin que la Universidad tenga que realizar un esfuerzo económico adicional contratando a profesorado específico para impartir asignaturas en inglés.

-Actividades propiamente dichas de formación en el conocimiento de inglés como competencia transversal. La Universidad de Jaén a este respecto ha realizado, entre otras, las siguientes: (1) la creación del Centro de Estudios Avanzados en Lenguas Modernas, con dos objetivos en lo que a nuestro estudio se refiere: (a) el establecimiento de un procedimiento para la acreditación del nivel lingüístico exigido en los nuevos planes de estudios ${ }^{3}$ y (b) la formación en idiomas; en un principio era restringida a alumnos y profesores (coordinadores académicos) que participaban en programas de movilidad, sin embargo en la actualidad se ha extendido a todos los

3 para la obtención del título de grado en todas las universidades andaluzas debe acreditarse el conocimiento de un segundo idioma, con un nivel Bl dentro del Marco Común de Referencia Europeo (CEFRL) (AUPA, 2008). 
miembros de la comunidad universitaria, lo que ha tenido una favorable acogida; y (2) la implantación de un programa de estancias puntuales en universidades americanas y británicas para la formación en inglés, dirigidas al profesorado y con financiación de la propia universidad.

Aunque entendemos que aún no es suficiente con estas actividades para conseguir un nivel de inglés que pueda otorgar ventajas competitivas significativas para el desarrollo profesional de nuestros egresados, sí creemos que se ha iniciado un camino sin retorno, en el que se seguirá avanzando de forma muy rápida en los próximos años. Con estos resultados se aporta evidencia sobre la necesidad de insistir en la formación para la adquisición de la competencia idiomática, lo que puede ser de una gran utilidad para los responsables universitarios en sus tareas de gobierno.

\section{BIBLIOGRAFÍA}

ANECA (2005): Libro Blanco del Título de Grado en Economía y en Empresa. Madrid: ANECA.

Aupa (Asociación de Universidades Públicas de Andalucía). Acta de 2 de mayo de 2008.

Bologna DeCLARATION (1999): The European Higher Education Area. Joint Declaration of the European Ministers of Education, convened in Bologna on 19 June 1999.

Corchuelo, M.B., Gallardo, D. y SánCHEZ, M.I. (2010): La percepción en las aulas universitarias de la inclusión de una lengua extranjera como experiencia de innovación didáctica. XX Jornadas Hispano-Lusas de Gestión Científica, Évora, Portugal.

EUROPEAN LANGUAGe COUNCIL (2001): Universities and language policy in Europe. Multilingualism and new learning environments. Berlín: Freie Universität Berlin.

GALLARDO, D., SÁNCHEZ, M.I. y CORCHUELO, B. (2008): La competencia transversal aportada por la inclusión de una lengua extranjera en los currícula de cara al Espacio Europeo de Educación superior. Jornada Nacional sobre estudios universitarios: de los proyectos de convergencia a la realidad de los nuevos títulos, Universidad Jaume I. Castellón de la Plana.

TUDOR, I. (2005): The challenge of the Bologna Process for Higher Education Language Teaching in Europe. Disponible en: http://userpage.fuberlin.de/ enlu/downloads/Bologna_ENLU_OK.rtf [09-04-2010]. 


\section{ANEXO 1. CUESTIONARIO SOBRE DOMINIO E IMPORTANCIA DEL INGLÉS AL LOS ALUMNOS CUESTIONARIO SOBRE DOMINIO E IMPORTANCIA DEL INGLÉS EN LA ENSEÑANZA UNIVERSITARIA.}

El presente cuestionario tiene como misión conocer el grado de dominio del idioma inglés así como la trascendencia de su conocimiento como segunda lengua dentro del alumnado universitario de la Licenciatura en Administración y Dirección de Empresas. Se encuadra dentro del Proyecto de Innovación Docente "El inglés como competencia transversal en el Espacio Europeo de Educación Superior: la Titulación de Administración y Dirección de Empresas" (PID 4A), concedido por el Vicerrectorado de Ordenación Académica, Innovación Docente y Profesorado de la Universidad de Jaén. Rogamos no deje sin contestar ninguna pregunta, pues ello imposibilitaría realizar un adecuado análisis posterior. Ningún cuestionario tendrá un tratamiento individualizado, siendo por tanto anónimo. Los datos serán custodiados por el equipo investigador que garantiza la absoluta confidencialidad de los mismos. Agradecemos el interés en la contestación del cuestionario y estamos a vuestra disposición en las siguientes direcciones: Francisca Castilla Polo, fpolo@ujaen.es; Alonso Moreno Aguayo, amoragu@ujaen.es; Macario Cámara de la Fuente, mcamara@ujaen.es; Eva Chamorro Rufián, echamo@ujaen.es

1. ¿ Qué lenguas conoce distintas a la materna? Indique en el espacio reservado cuál o cuáles son.

Centrándonos a partir de ahora en la lengua o idioma inglés,

\begin{tabular}{|l|l|}
\hline 1. & \\
\hline 2. & \\
\hline 3. & \\
\hline
\end{tabular}
responda a las siguientes cuestiones

2. Aparte de la enseñanza reglada en educación primaria y secundaria, ¿̇qué tiempo le ha dedicado a su aprendizaje? (años):

\begin{tabular}{|l|l|}
\hline Estudio de inglés por cuenta propia & \\
\hline Estudios de inglés en Academia de Idiomas & \\
\hline Estudios de inglés en Escuelas Oficiales de & \\
Idiomas. & \\
\hline Otro tipo de estudios (Especificar) & \\
\hline
\end{tabular}

3. ¿Ha realizado alguna actividad de movilidad en el extranjero?

En caso afirmativo, especifique el tipo de actividad, duración y destino en la siguiente tabla (señale con una $X$ ):

\begin{tabular}{|l|l|l|l|l|}
\hline $\begin{array}{l}\text { Tipo de } \\
\text { Actividad }\end{array}$ & $\begin{array}{l}\text { Estudios pre- } \\
\text { universitarios } \\
\text { en el } \\
\text { extranjero }\end{array}$ & $\begin{array}{l}\text { Estancias de } \\
\text { verano en el } \\
\text { extranjero }\end{array}$ & $\begin{array}{l}\text { Movilidad } \\
\text { Erasmus }\end{array}$ & Otros (especificar) \\
\hline Realización & & & & \\
\hline $\begin{array}{l}\text { Lugar } \\
\text { (ciudad, país) }\end{array}$ & & & & \\
\hline $\begin{array}{l}\text { Duración } \\
\text { (meses) }\end{array}$ & & & & \\
\hline
\end{tabular}


En caso negativo, especifique los motivos (señale con una X).

\begin{tabular}{|l|l|}
\hline No lo considero necesario & \\
\hline Insuficiente nivel de inglés & \\
\hline Motivos de tipo económico & \\
\hline Motivos de tipo familiar/personal & \\
\hline Otros (Especificar) & \\
\hline
\end{tabular}

4. ¿Posee algún certificado de reconocimiento de su nivel de inglés? Si No (señale con un círculo la respuesta). Sólo en caso afirmativo especifique cuál o cuáles en la siguiente tabla.

\begin{tabular}{|l|l|}
\hline Certificado de la Escuela Oficial de Idiomas (Nivel intermedio $-3^{\circ}$ curso) & \\
\hline Certificado de la Escuela Oficial de Idiomas (Nivel superior $-5^{\circ}$ curso) & \\
\hline Toefl (indique puntuación obtenida) & \\
\hline Cambridge: First Certificate & \\
\hline Cambridge: Advanced Certificate & \\
\hline Cambridge: Proficiency Certificate & \\
\hline Otro (Especificar) & \\
\hline
\end{tabular}

5. ¿Qué dominio considera que posee de la lengua inglesa? (señale con una X):

\begin{tabular}{|l|l|l|l|l|l|}
\hline & Muy bajo & Bajo & Medio & Alto & Muy alto \\
\hline Lectura & & & & & \\
\hline Escritura & & & & & \\
\hline Conversación & & & & & \\
\hline
\end{tabular}

6. ¿Sería capaz de preparar un trabajo corto (resolución de un caso, opinión justificada sobre un tema, etc...) en inglés? Si No (señale con un círculo la respuesta). En caso afirmativo, indique la calidad esperada:

\begin{tabular}{|l|c|c|c|c|c|}
\hline & Muy baja & Baja & Regular & Alta & Muy alta \\
\hline SI & & & & & \\
\hline
\end{tabular}

7. ¿Sería capaz de exponer en inglés un trabajo corto a sus compañeros en una clase ayudado de soporte informático (power point o similar)? Si No (señale con un círculo la respuesta). En caso afirmativo, indique la calidad esperada:

\begin{tabular}{|l|l|l|l|l|c|}
\hline & Muy baja & Baja & Regular & Alta & Muy alta \\
\hline SI & & & & & \\
\hline
\end{tabular}

8. ¿Sería capaz de seguir la exposición realizada en inglés por otros compañeros en clase? Si No (señale con un círculo la respuesta). En caso afirmativo, indique la calidad esperada:

\begin{tabular}{|l|c|c|c|c|c|}
\hline & Muy baja & Baja & Regular & Alta & Muy alta \\
\hline SI & & & & & \\
\hline
\end{tabular}

9. Si un profesor imparte una clase en inglés, ¿considera que podría entenderlo? Si No (señale con un círculo la respuesta). En caso afirmativo, indique la calidad esperada:

\begin{tabular}{|l|l|l|l|l|c|}
\hline & Muy baja & Baja & Regular & Alta & Muy alta \\
\hline SI & & & & & \\
\hline
\end{tabular}


10. Si el profesor le facilitara bibliografía (temas, artículos para lectura, ...) en inglés, ¿le resultaría fácil su lectura y comprensión? Si No (señale con un círculo la respuesta). En caso afirmativo, indique la calidad esperada.

\begin{tabular}{|l|c|c|c|c|c|}
\hline & Muy baja & Baja & Regular & Alta & Muy alta \\
\hline SI & & & & & \\
\hline
\end{tabular}

11. ¿Considera necesario mejorar su dominio de la lengua inglesa? Si No (señale con un círculo la respuesta). En caso afirmativo indique el grado de mejora necesitado:

\begin{tabular}{|l|l|l|l|}
\hline & Bajo & Medio & Elevado \\
\hline SI & & & \\
\hline
\end{tabular}

12. ¿Cómo abordaría, en caso de que lo considere necesario, la mejora de su dominio de la lengua inglesa? Señala con una X la opción/opciones que más oportunas le parezcan.

\begin{tabular}{|l|l|}
\hline Estudio de inglés por cuenta propia & \\
\hline Estudios de inglés en Academia de Idiomas & \\
\hline Estudios de inglés en Escuelas Oficiales de Idiomas. & \\
\hline Estancia en el extranjero & \\
\hline Otro tipo de estudios (Especificar) & \\
& \\
\hline
\end{tabular}

13. Valore el grado de importancia del idioma inglés como segunda lengua en la mejora de sus oportunidades laborales.

\begin{tabular}{|l|l|l|l|l|}
\hline Muy bajo & Bajo & Medio & Alto & Muy alto \\
\hline & & & & \\
\hline
\end{tabular}

14. Valore el grado de importancia del idioma inglés para los distintos destinos del mercado laboral a los que puede acceder con su Titulación universitaria.

\begin{tabular}{|l|l|l|l|l|l|}
\hline & Muy bajo & Bajo & Medio & Alto & Muy alto \\
\hline $\begin{array}{l}\text { Trabajo en entidades } \\
\text { y/o organizaciones } \\
\text { públicas }\end{array}$ & & & & & \\
\hline $\begin{array}{l}\text { Trabajo en empresas } \\
\text { privadas }\end{array}$ & & & & & \\
\hline $\begin{array}{l}\text { Trabajo en } \\
\text { Universidades }\end{array}$ & & & & & \\
\hline
\end{tabular}

15. ¿Considera que el idioma inglés debería estar presente a lo largo de la formación universitaria? Si No (señale con un círculo la respuesta). En caso afirmativo valore la necesidad de su incorporación:

\begin{tabular}{|l|c|c|c|c|c|}
\hline & Muy baja & Baja & Regular & Alta & Muy alta \\
\hline SI & & & & & \\
\hline
\end{tabular}

16. ¿Estaría interesado en recibir alguna asignatura en una lengua inglesa?:

\begin{tabular}{|c|c|}
\hline SI & NO \\
\hline & \\
\hline
\end{tabular}


Explique muy brevemente el por qué de su respuesta

Sólo en caso afirmativo, ¿qué tipo de asignatura considera que se adaptaría mejor a la utilización del inglés como idioma? Señale con una $X$.

\begin{tabular}{|l|c|c|}
\hline $\begin{array}{l}\text { Asignatura } \\
\text { Obligatoria }\end{array}$ & $\begin{array}{c}\text { Asignatura } \\
\text { Troncal }\end{array}$ & $\begin{array}{c}\text { Asignatura } \\
\text { Optativa }\end{array}$ \\
\hline & & \\
\hline
\end{tabular}

17. ¿Qué grado de importancia le daría al hecho de contar en su proceso de formación una asignatura de este tipo?:

\begin{tabular}{|c|c|c|c|c|}
\hline Muy baja & Baja & Regular & Alta & Muy alta \\
\hline & & & & \\
\hline
\end{tabular}

Indique por favor el curso al que pertenece (señala con una X; en caso de pertenecer a varios, señale el curso más alto cursado).

\begin{tabular}{|l|l|l|l|}
\hline $1^{\circ}$ & $2^{\circ}$ & $3^{\circ}$ & $4^{\circ}$ \\
\hline
\end{tabular}

Si usted es actualmente un estudiante extranjero Erasmus en España, por favor indique su país de procedencia:

Muchas gracias por su colaboración 


\section{ANEXO 2. CUESTIONARIO SOBRE DOMINIO E IMPORTANCIA DEL INGLÉS A LOS PROFESORES CUESTIONARIO SOBRE DOMINIO E IMPORTANCIA DEL INGLÉS EN LA ENSEÑANZA UNIVERSITARIA.}

1. ¿Qué lenguas conoce distintas a la materna? Indique en el espacio reservado cuál o cuáles son.

\begin{tabular}{|l|l|}
\hline 1. & \\
\hline 2. & \\
\hline 3. & \\
\hline
\end{tabular}

Centrándonos a partir de ahora en la lengua o idioma inglés, responda a las siguientes cuestiones

2. Aparte de la enseñanza reglada en educación primaria y secundaria, ¿qué tiempo le ha dedicado a su aprendizaje? (años):

Estudio de inglés por cuenta propia

Estudios de inglés en Academia de Idiomas

Estudios de inglés en Escuelas Oficiales de Idiomas.

Otro tipo de estudios (Especificar)

3. ¿Ha realizado alguna actividad de movilidad en el extranjero?

En caso afirmativo, especifique el tipo de actividad, duración y destino en la siguiente tabla.

\begin{tabular}{|l|c|c|c|c|c|c|c|}
\hline $\begin{array}{l}\text { Tipo de } \\
\text { Actividad }\end{array}$ & $\begin{array}{c}\text { Estudios pre- } \\
\text { universitarios } \\
\text { en el } \\
\text { extranjero }\end{array}$ & $\begin{array}{c}\text { Estancias } \\
\text { de verano } \\
\text { en el } \\
\text { extranjero }\end{array}$ & $\begin{array}{c}\text { Movilidad } \\
\text { Erasmus }\end{array}$ & $\begin{array}{c}\text { Visitas } \\
\text { Docentes }\end{array}$ & $\begin{array}{c}\text { Estancias } \\
\text { pre- } \\
\text { doctorales }\end{array}$ & $\begin{array}{c}\text { Estancias } \\
\text { post- } \\
\text { doctorales }\end{array}$ & $\begin{array}{c}\text { Otros } \\
\text { (especificar) }\end{array}$ \\
\hline $\begin{array}{l}\text { Lugar } \\
\text { (ciudad, } \\
\text { país) }\end{array}$ & & & & & & & \\
\hline $\begin{array}{l}\text { Duración } \\
\text { (meses) }\end{array}$ & & & & & & & \\
\hline
\end{tabular}

En caso negativo, especifique los motivos (señale con una $\mathrm{X}$ ).

\begin{tabular}{|l|l|}
\hline No lo considero necesario & \\
\hline Insuficiente nivel de inglés & \\
\hline Motivos de tipo económico & \\
\hline Motivos de tipo familiar/personal & \\
\hline Otros (Especificar) & \\
& \\
\hline
\end{tabular}

4. ¿Posee algún certificado de reconocimiento de su nivel de inglés? Si No (señale con un círculo la respuesta). Sólo en caso afirmativo especifique cuál o cuáles en la siguiente tabla.

\begin{tabular}{|l|l|}
\hline Certificado de la Escuela Oficial de Idiomas (Nivel intermedio $-3^{\circ}$ curso) & \\
\hline Certificado de la Escuela Oficial de Idiomas (Nivel superior $-5^{\circ}$ curso) & \\
\hline Toefl (indique puntuación obtenida) & \\
\hline Cambridge: First Certificate & \\
\hline Cambridge: Advanced Certificate & \\
\hline Cambridge: Proficiency Certificate & \\
\hline Otro (Especificar) & \\
\hline
\end{tabular}


Francisca Castilla, Alonso Moreno, Macario Cámara, Eva $M^{\circ}$ Chamorro

Competencia del inglés en los universitarios de empresa

5. ¿Qué dominio considera que posee de la lengua inglesa? (señale con una X):

\begin{tabular}{|l|l|l|l|l|l|}
\hline & Muy bajo & Bajo & Medio & Alto & Muy alto \\
\hline Lectura & & & & & \\
\hline Escritura & & & & & \\
\hline Conversación & & & & & \\
\hline
\end{tabular}

6. ¿Sería capaz de exponer en inglés sus clases teóricas y prácticas ayudado de soporte informático (power point o similar)? Si No (señale con un círculo la respuesta). En caso afirmativo, indique la calidad esperada:

\begin{tabular}{|l|l|l|l|l|c|}
\hline & Muy baja & Baja & Regular & Alta & Muy alta \\
\hline SI & & & & & \\
\hline
\end{tabular}

7. ¿Participa actualmente en el Programa P.A.T.I.E. organizado por el Vicerrectorado de Relaciones Internacionales de la Universidad de Jaén? Si No (señale con un círculo la respuesta). En caso afirmativo, indique el nivel ofrecido para su respectiva asignatura o asignaturas:

\begin{tabular}{|l|l|l|l|}
\hline & Bajo & Medio & Elevado \\
\hline SI & & & \\
\hline
\end{tabular}

8. ¿Está actualmente impartiendo algún contenido, aunque sea mínimo, en idioma inglés para sus alumnos fuera del Programa P.A.T.I.E.? Si No (señale con un círculo la respuesta).

9. ¿Está facilitando bibliografía en idioma inglés para asignaturas no incluidas en el Programa P.A.T.I.E.? Si No (señale con un círculo la respuesta).

10. ¿Considera necesario mejorar su dominio de la lengua inglesa? Si No (señale con un círculo la respuesta). En caso afirmativo indique el grado de mejora necesitado:

\begin{tabular}{|l|l|l|l|}
\hline & Bajo & Medio & Elevado \\
\hline SI & & & \\
\hline
\end{tabular}

11. ¿Cómo abordaría, en caso de que lo considere necesario, la mejora de su dominio de la lengua inglesa? Señala con una X la opción/opciones que más oportunas le parezcan.

\begin{tabular}{|l|l|}
\hline Estudio de inglés por cuenta propia & \\
\hline Estudios de inglés en Academia de Idiomas & \\
\hline Estudios de inglés en Escuelas Oficiales de Idiomas. & \\
\hline Estancia en el extranjero & \\
\hline Otro tipo de estudios (Especificar) & \\
\hline
\end{tabular}

12. Valore el grado de importancia del idioma inglés como segunda lengua en la mejora de sus oportunidades laborales.

\begin{tabular}{|c|c|c|c|c|}
\hline Muy bajo & Bajo & Medio & Alto & Muy alto \\
\hline & & & & \\
\hline
\end{tabular}

13. ¿Considera que el idioma inglés debería estar presente a lo largo de la formación universitaria? Si No (señale con un círculo la respuesta). En caso afirmativo valore la necesidad de su incorporación:

\begin{tabular}{|l|l|l|l|l|l|}
\hline & Muy baja & Baja & Regular & Alta & Muy alta \\
\hline SI & & & & & \\
\hline
\end{tabular}


14. ¿Estaría interesado en impartir alguna asignatura en una lengua inglesa?:

\begin{tabular}{|l|l|}
\hline SI & NO \\
\hline & \\
\hline
\end{tabular}

Explique muy brevemente el por qué de su respuesta

Sólo en caso afirmativo, ¿qué tipo de asignatura considera que se adaptaría mejor a la utilización del inglés como idioma? Señale con una $X$.

\begin{tabular}{|c|c|c|}
\hline $\begin{array}{c}\text { Asignatura } \\
\text { Obligatoria }\end{array}$ & $\begin{array}{c}\text { Asignatura } \\
\text { Troncal }\end{array}$ & $\begin{array}{c}\text { Asignatura } \\
\text { Optativa }\end{array}$ \\
\hline & & \\
\hline
\end{tabular}

15. ¿Qué grado de importancia le daría al hecho de que los alumnos contaran en su proceso de formación una asignatura de este tipo?

\begin{tabular}{|l|l|l|l|l|}
\hline Muy baja & Baja & Regular & Alta & Muy alta \\
\hline & & & & \\
\hline
\end{tabular}

16. Indica por favor el curso/cursos donde impartes docencia dentro de la titulación Administración y Dirección de Empresas:

\begin{tabular}{|l|l|l|l|}
\hline $1^{\circ}$ & $2^{\circ}$ & $3^{\circ}$ & $4^{\circ}$ \\
\hline
\end{tabular}

17. Te agradeceríamos que nos indicases los siguientes datos profesionales y académicos:

a) Categoría

laboral:

b) Titulación académica (señale con una X)

\begin{tabular}{|l|l|}
\hline Ciencias Empresariales, Económicas o Administración y Dirección de & \\
Empresas & \\
\hline Derecho & \\
\hline Estadística/Matemáticas & \\
\hline Otros (Especificar) & \\
\hline
\end{tabular}

Muchas gracias por su colaboración 


\section{ANEXO 3. CUESTIONARIO SOBRE COMPRENSIÓN DEL INGLÉS A LOS ALUMNOS CUESTIONARIO SOBRE COMPRENSIÓN DE INGLÉS EN LA ENSEÑANZA UNIVERSITARIA.}

El presente cuestionario tiene como misión conocer el grado de comprensión y entendimiento del idioma inglés como segunda lengua dentro del alumnado universitario de la Licenciatura en Administración y Dirección de Empresas. Se encuadra dentro del Proyecto de Innovación Docente "El inglés como competencia transversal en el Espacio Europeo de Educación Superior: la Titulación de Administración y Dirección de Empresas" (PID 4A), concedido por el Vicerrectorado de Ordenación Académica, Innovación Docente y Profesorado de la Universidad de Jaén. Rogamos no deje sin contestar ninguna pregunta, pues ello imposibilitaría realizar un adecuado análisis posterior. Ningún cuestionario tendrá un tratamiento individualizado, siendo por tanto anónimo. Los datos serán custodiados por el equipo investigador que garantiza la absoluta confidencialidad de los mismos. Agradecemos el interés en la contestación del cuestionario y estamos a vuestra disposición en las siguientes direcciones: Francisca Castilla Polo, fpolo@ujaen.es; Alonso Moreno Aguayo, amoragu@vjaen.es; Macario Cámara de la Fuente, mcamara@ujaen.es; Eva Chamorro Rufián, echamo@ujaen.es

1. Tras la clase impartida en inglés, ¿̇considera que ha entendido su contenido? Si No (señale con un círculo la respuesta). En caso afirmativo, indique el grado de comprensión alcanzado.

\begin{tabular}{|l|l|l|l|l|l|}
\hline & Muy bajo & Bajo & Regular & Alto & Muy alto \\
\hline SI & & & & & \\
\hline
\end{tabular}

2. Tras la clase impartida en inglés, ¿̇considera necesitaría mejorar su dominio del idioma inglés? Si No (señale con un círculo la respuesta). En caso afirmativo, indique el grado necesitado para cada uno de los siguientes aspectos.

\begin{tabular}{|l|l|l|l|l|l|}
\hline & Muy bajo & Bajo & Medio & Alto & Muy alto \\
\hline Lectura & & & & & \\
\hline Escritura & & & & & \\
\hline Conversación & & & & & \\
\hline
\end{tabular}

3. Conteste a las siguientes preguntas sobre el contenido de la clase impartida en inglés:

1. ¿̇Es Alemania un país exportador neto?

2. El concepto de importación depende de dónde se gasta el dinero y no de dónde viene el dinero ¿̀verdadero o falso?

3. ¿De qué país tomó EEUU dinero prestado para la Guerra de Irak?

4. ¿ ¿uál ha sido el acontecimiento económico más importante para España de los últimos 50 años según el ponente?

5. ¿Qué país hay completamente libre de restricciones comerciales?

6. ¿Qué industria americana de ocio sufre restricciones por parte de Francia?

7. ¿Cuál es la opinión del ponente sobre las restricciones al comercio? 